\title{
Pinhole defects in nitrile gloves
}

\author{
A preliminary report on the incidence of pre-existing pinhole defects in nitrile dental gloves
}

H. B. Patel, G. J. P. Fleming and F. J. Trevor Burke Br Dent J 2003; 195: 509-512

Introduction

Examination gloves manufactured from natural latex have been the predominant glove choice to date in dental practice. However, concerns over hypersensitivity have resulted in the use of alternatives such as nitrile gloves. The aim of the current study was to assess the incidence of pre-existing pinhole defects in nitrile examination gloves.

\section{Methods}

Air inflation, followed by water submersion, was used to assess the incidence of pre-existing pinhole defects in five nitrile and two latex glove types. The gloves were filled with a constant volume of air and submerged in 3 litres of water for 10 seconds while being observed for air bubbles which would indicate pinhole defects. The position and number of pinholes were noted for 100 gloves of each type investigated.

Results

The incidence of pre-existing pinholes for latex gloves was 0\% for the non-sterile surgical latex glove type and 3\% for the powdered latex examination glove type, with pinholes located on the thumb, middle finger and ring finger. Of the nitrile gloves evaluated, three types were assessed to have no pre-existing pinhole defects. One type had a $2 \%$ incidence of pre-existing pinhole defects - one pinhole located on the thumb region of the glove and one on the ring finger portion of the glove. The fifth nitrile glove type had one pre-existing pinhole defect located on the middle finger.

\section{Significance}

All glove types examined met the European Standard (EN 455-1) and there was no statistically significant difference between glove types. However, the nitrile gloves generally exhibited less preexisting pinhole defects than the latex examination gloves.

\section{IN BRIEF}

- Defects in nitrile gloves prior to use may not be significantly different in number from natural latex gloves.

- Nitrile gloves may provide satisfactory barrier protection and could be of value to dental healthcare workers (DHCWs) who have allergies to natural rubber latex.

\section{COMMENT}

Within dental practice, gloves that provide an effective barrier of protection from infectious organisms are a prerequisite for the protection of both patients and dental personnel. Members of the dental profession wear gloves far longer than any other healthcare provider, culminating in 10 glove wearing years over the average working lifetime. To date, natural rubber latex (NRL) has consistently been the most satisfactory raw material for the manufacture of gloves. However, certain latex proteins inherent within the material may pose a risk of provoking allergic reactions in patients and healthcare workers. The prevalence of latex sensitisation in health care workers has been shown to vary between $0 \%$ and $30 \%$ and the causal relationship between the use of latex gloves and hypersensitivity is well documented.

In response to this increase in latex allergies, gloves manufactured from a variety of non-latex materials have been introduced into the healthcare domain. Published studies have demonstrated that both latex and nitrile gloves provide comparable barrier protective qualities in both laboratory and clinical use. Glove quality can vary greatly between manufacturers, consequently not all gloves are capable of the same degree of protection. Indeed, concerns have been raised of possible higher levels of punctures present within unused nitrile gloves in comparison with unused latex gloves.

This study by Hetal Patel and colleagues addresses this matter, comparing the incidence of pre-existing pinhole defects in more recently introduced nitrile examination gloves (500 gloves, five brands) with latex glove types (200 gloves, two brands). The authors used an air inflation method followed by a water submersion technique that is comparable with the European standard for assessing pre-existing pinholes. They ascertained that the nitrile gloves examined exhibited less pre-existing pinhole defects than the latex glove brands tested though this was not statistically significant. They conclude that nitrile gloves are a suitable replacement to latex dependant upon clinical comfort.

This timely study reassures that current manufacturing processes continue to improve the quality of nitrile gloves, which now appear to be an appropriate alternative to latex. Whilst it may have previously been thought that the provision of a completely latexfree setting in dental surgeries was unrealistic, this objective becomes ever more attainable. Maximum effort should be made to minimize the unnecessary exposure of both patients and healthcare workers to latex allergens in this high-risk environment and the routine use of non-latex gloves would be a significant step forward.

Colin A. Murray, Clinical Lecturer in Restorative Dentistry, University of Glasgow Dental School and Hospital doi:10.1038/sj.bdj.4810665 